Mens

revue d'histoire intellectuelle de l'Amérique française

MENS

\title{
Isolement et rencontre chez quatre lectrices québécoises d'avant 1960
}

\section{Elsa Pépin}

Volume 5, numéro 2, printemps 2005

L'histoire du livre au Québec, de la Nouvelle-France au XX ${ }^{\mathrm{e}}$ siècle

URI : https://id.erudit.org/iderudit/1024362ar

DOI : https://doi.org/10.7202/1024362ar

Aller au sommaire du numéro

Éditeur(s)

Centre de recherche en civilisation canadienne-française

ISSN

1492-8647 (imprimé)

1927-9299 (numérique)

Découvrir la revue

Citer cet article

Pépin, E. (2005). Isolement et rencontre chez quatre lectrices québécoises d'avant 1960. Mens, 5(2), 465-496. https://doi.org/10.7202/1024362ar

\section{Résumé de l'article}

Dans le cadre d'une analyse des lectures de jeunesse chez quatre autobiographes québécoises nées dans les années 1910-1920, le rôle déterminant de la censure du livre s'est avéré révélateur d'une dynamique propre au changement progressif des mentalités à l'époque. En effet, la relation des quatre jeunes lectrices avec la contrainte et l'interdit de lecture témoigne des problématiques liées à la condition de l'individu et de la femme par rapport à l'autoritarisme du clergé, au didactisme de l'enseignement, à la limitation de l'espace de liberté caractérisée par la présence de l'Index par exemple. À travers la lecture clandestine, ces femmes revendiquent une liberté de penser et partent à la conquête du monde, mais elles subissent également un isolement problématique, fondamental pour la constitution de leur identité et de leur engagement social. 


\title{
ISOLEMENT ET RENCONTRES CHEZ QUATRE LECTRICES QUÉBÉCOISES D'AVANT 1960
}

\author{
Elsa Pépin \\ Département de langue et littérature françaises \\ Université McGill \\ elsa_pepin@yahoo.com
}

\section{Résumé}

Dans le cadre d'une analyse des lectures de jeunesse chez quatre autobiographes québécoises nées dans les années 1910-1920, le rôle déterminant de la censure du livre s'est avéré révélateur d'une dynamique propre au changement progressif des mentalités à l'époque. En effet, la relation des quatre jeunes lectrices avec la contrainte et l'interdit de lecture témoigne des problématiques liées à la condition de l'individu et de la femme par rapport à l'autoritarisme du clergé, au didactisme de l'enseignement, à la limitation de l'espace de liberté caractérisée par la présence de l'Index par exemple. À travers la lecture clandestine, ces femmes revendiquent une liberté de penser et partent à la conquête du monde, mais elles subissent également un isolement problématique, fondamental pour la constitution de leur identité et de leur engagement social.

\section{Abstract}

Through the analysis of the youth readings of four Quebec autobiographical writers born in the 1910s and 1920s, the determinative role of book censorship was found to be indicative of a progressive change of mentality in that period. For instance, the relationship of the four young women readers with the various restrictions and probibitions surrounding reading reveals a number of issues related to the condition of the individual and of women with regards to the authoritarianism of the clergy, the didacticism of education, and the limitations 
of freedom characterised by the Index. Through clandestine readings, these women demanded freedom of thought and set out to conquer the world, but they also suffered from problematical isolation that was fundamental to the constitution of their identity and to their social commitment.

Les obstacles et la censure ont toujours stimulé les mauvaises têtes et ne les ont pas empêchées, en tous cas, d'explorer les mondes interdits'.

Afin de saisir la dimension individuelle de l'histoire du livre, il faut interroger les lecteurs, acteurs essentiels dans le réseau qui unit les institutions, les écrivains, les éditeurs, mais également chacun de ceux qui consomment le livre. L'histoire de la lecture s'impose donc comme un pan essentiel, mais aussi complexe, des études consacrées au livre, à son histoire et à sa représentation. Cependant, les chercheurs font face à plusieurs écueils, suscités par la nature fuyante, plurivoque et " hybride » de la notion de lecture et par l'étendue et l'hétérogénéité de ses sources. Dans leur étude, Yvan Lamonde et Sophie Montreuil ont offert une définition de la lecture comme "rencontre physique et intellectuelle d'un objet, le livre, et d'un individu, le lecteur ${ }^{2}$ ", insistant sur cette relation fondamentale qui unit l'individu à l'objet, susceptible d'être décrite et analysée. Parmi les sources documentaires qui s'offrent à l'historien de la lecture, les témoignages individuels, soit l'autobiographie, les mémoires, le journal intime et les souvenirs, forment un ensemble particulièrement intéressant qui offre une perspective singulière de l'expérience de lecture, tout en faisant de la lecture un phénomène fondamentalement pluriel, à l'image de la pluralité des êtres et des perceptions de lecture. Bon nombre d'historiens du livre ont d'ailleurs noté la particularité de ces sources individuelles ${ }^{3}$, 
qui demandent cependant à être manipulées avec la plus grande prudence, car l'interprétation de ces documents doit se conjuguer au problème de leur représentativité. Il semble essentiel d'éclairer les pratiques de lecture répertoriées dans la littérature personnelle à la lumière du contexte socio-historique, selon l'environnement social et culturel qui les produit et en rapport avec la communauté à laquelle l'individu appartient ${ }^{4}$. Cependant, leur singularité doit demeurer primordiale à leur compréhension : l'expérience de lecture décrite doit représenter la différence selon les individus plutôt que le phénomène de lecture en général ${ }^{5}$.

Cette étude se penche donc sur les pratiques de lecture de quatre jeunes filles québécoises nées entre 1914 et 1926, soit Claire Martin, Simonne Monet-Chartrand, Paule SaintOnge et Thérèse Lesage-Vézina, qui ont toutes écrit une autobiographie où la lecture occupe un rôle fondamental, avec une attention particulière portée à la variabilité de l'expérience individuelle dans le contexte d'une même communauté, soit le Québec d'avant $1960^{\circ}$. Rapidement, il faut présenter ces quatre jeunes femmes qui ont toutes un parcours assez différent, mais qui se rejoignent dans leur rapport à l'interdit qui marque leur découverte de la lecture. Claire Martin, née en 1914 dans une famille pauvre montréalaise et orpheline de mère très jeune, subit l'autorité tyrannique d'un père cruel et des sœurs enseignantes. Pour sa part, Simonne MonetChartrand, née à Montréal en 1919, vient d'un milieu bourgeois et libéral qui la stimule et l'oriente dans sa lecture, tandis que Paule Saint-Onge, née en 1922, grandit à Montréal dans un milieu pauvre et inculte, où elle doit affronter le comportement d'une mère irrationnelle. Finalement, la quatrième lectrice de notre corpus, Thérèse Lesage-Vézina, la seule originaire d'un milieu rural, est née en 1926 à Pont-Rouge, dans la région de Québec. Orpheline très jeune, Thérèse Lesage- 
Vézina est élevée par une marraine qui la guide dans ses lectures, mais elle n'a pas accès à autant de livres que les enfants de Montréal.

Ces quatre lectrices ont été sélectionnées parmi un corpus de littérature personnelle parce que, d'une part, elles font toutes parties d'une époque bien définie (nées entre 1914 et 1926) et, que, d'autre part, leurs autobiographies respectives se penchent sur le rôle déterminant de la lecture dans leur jeunesse et évoquent l'impact majeur qu'a joué l'interdit dans la formation de leur identité. De plus, ces quatre exemples illustrent des cas relativement représentatifs : deux jeunes filles de milieux pauvres, une Montréalaise d'un milieu bourgeois et une fille originaire d'un milieu rural. Leurs textes offrent donc des témoignages assez variés sur l'expérience personnelle de lecture, tout en ciblant une problématique plus généralisée, celle du rapport à la censure.

L'autobiographie, privilégiée dans cette étude au détriment du journal intime ou des mémoires, s'est avérée le genre personnel le plus fécond pour l'analyse des pratiques de lecture, analysées de manière plus approfondies grâce à la rétrospection que permet ce genre. L'autobiographie semble en effet constituer une source documentaire idéale qui révèle la particularité de la relation de l'individu au livre et offre un témoignage précieux pour l'histoire des mentalités. Philippe Lejeune a déjà démontré que dans le genre de l'autobiographie, la lecture représente souvent une phase initiatique dans la formation de l'identité. De plus, la lecture constitue un objet privilégié de l'autobiographie car en tant qu'activité qui engage l'être vers l'autre et vers le monde, la lecture délimite l'espace de soi. Il ne faut cependant pas perdre de vue le fait que l'être qui se présente dans l'autobiographie est un être de papier qui ne reflète pas la réalité en tous points. Il s'agit en effet d'une construction, et les pratiques de lecture ici pré- 
sentées sont des représentations qui ne peuvent pas être tenues comme des manifestations du réel.

Parmi les diverses problématiques suscitées par l'étude de la lecture dans notre corpus, celle du rapport de l'enfant face à la censure est apparue particulièrement révélatrice pour la compréhension des pratiques de lecture de l'époque et de l'évolution des mentalités. En effet, la plupart des textes du corpus consacrent explicitement une section à la description des lectures d'enfance et de jeunesse, celles-ci occupant visiblement un jalon important dans la formation de l'identité des autobiographes, et s'attardent au rapport complexe avec une censure omniprésente et étouffante. L'analyse des différentes dynamiques de lecture instaurées durant la jeunesse et influencées par la présence d'une norme vient dévoiler la spécificité des expériences individuelles de lecture qui répondent différemment à un même interdit social.

La censure du livre au Québec dans les années 19201960 connait d'importantes mutations, relevées par un des spécialistes de la question, Pierre Hébert $^{8}$, qui explique l'éclatement du sujet censeur et des manifestations de la censure durant cette période qualifiée de "zone de transition cruciale » qui correspond au "passage d'une censure affichée et légitimée à une autre aux contours moins définis ${ }^{9} »$. La censure deviendrait donc moins visible, se faisant indirecte et plus insidieuse. Sans prétendre divulguer de manière systématique les effets de cette forme de censure plus diffuse, plurielle et souterraine, il paraît grandement intéressant d'examiner à travers les autobiographies de cette période comment se vit la censure de l'intérieur, éclairée par la vision de l'autobiographe qui remonte aux origines du " moi » et qui tente de comprendre un cheminement personnel. À travers l'analyse de l'impact de la censure sur la manière de lire de ces jeunes lectrices, se dessine un tableau moral et psychologique de la lec- 
trice confrontée à la dynamique censoriale et avide de se libérer d'une société peu prompte à encourager l'épanouissement des jeunes filles. En effet, l'étude de la lecture chez ces quatre écrivaines permet l'analyse des balbutiements de la lutte de la femme québécoise pour accéder aux droits et libertés, dont le livre offre un premier accès.

Cette étude se fait en deux temps, suivant les deux pôles de la censure, soit sa forme prescrite et sa forme proscrite. La première partie porte sur la prescription, soit sur la censure comme "obligation", tandis que la seconde partie de l'analyse se penche sur la proscription, soit sur la censure comme " interdiction ", " comme si empêcher de dire et fixer les conditions de la parole représentait les deux faces d'un même pouvoir de contrainte ${ }^{10} »$.

\section{Lecture d'enfance et de jeunesse prescrites ou les limites d'une lecture sous tutelle}

L'étude des lectures d'enfance et de jeunesse est particulièrement éclairante pour comprendre la relation qui s'instaure entre l'individu et le livre, relation examinée ainsi depuis son origine. Soumises à un contrôle insistant du clergé, les lectures d'enfance des quatre femmes du corpus se caractérisent par une forte prescription et s'inscrivent dans une dynamique particulière avec l'autorité. Bien que l'imposition des lectures aux enfants soit un phénomène fréquent, le choix des livres peut être plus ou moins limité selon l'étroitesse ou la largesse d'esprit des prescripteurs.

Thérèse Lesage-Vézina réfléchit longuement à la nature des lectures prescrites de son enfance, peut-être faute d'avoir eu accès à d'autres lectures, mais aussi parce que toute son autobiographie se construit autour des livres, véritables jalons de son existence. À cet égard, il faut noter que son témoignage offre aux historiens du livre une source particu- 
lièrement privilégiée, mais peut-être peu représentative car anormalement abondante en références aux livres. Citant Denise Escarpit, Lesage-Vézina établit un parallèle intéressant entre la littérature didactique européenne et la littérature prescrite qui prévaut au Québec dans les années quarante : "Aussi la littérature didactique eut-elle un double aspect: la littérature pédagogique directement utilitaire (livres scolaires) et la littérature morale (fables, contes, etc.) ou religieuse (vies des saints, exempla) ${ }^{11}$." En effet, cette classification se rapproche sensiblement des lectures de jeunesse prescrites chez les femmes du corpus et l'aspect didactique se révèle caractéristique. Ces lectures se distribuent dans les textes du corpus en deux catégories, selon qu'elles sont effectuées à l'école ou à la maison.

À l'égard des manuels scolaires, les commentaires des quatre autobiographes révèlent une forme d'insatisfaction, apparemment caractéristique de leur rapport avec les livres d'école. En ce qui concerne l'apprentissage de la littérature, le manuel d'histoire littéraire auquel font référence les jeunes filles du corpus, qui fréquentent toutes le couvent, est écrit par les Frères de l'instruction chrétienne. Ce manuel contient des « extraits de chefs-d'œuvre de la littérature française et canadienne où une large part [est] faite aux écrivains catholiques et à certains autres ayant acquis une notoriété littéraire ${ }^{12}$ ", selon la description de Lesage-Vézina. Elle y découvre par exemple Pamphile Le May, pionnier de la poésie canadiennefrançaise, et Mme de Sévigné, pour qui elle développera une réelle admiration. Plutôt satisfaite de ce manuel, LesageVézina a le privilège de pouvoir retrouver dans la bibliothèque de sa tante adoptive les livres évoqués par son livre scolaire.

Pour sa part, Paule Saint-Onge considère que « les Frères des écoles chrétiennes qui avaient produit ce manuel 
avaient dressé des autels à un tas de bonshommes sans importance $[\ldots]$ et $[. .$.$] avaient ignoré ou vilipendé presque tous$ les écrivains de talent ${ }^{13}$. » Le manuel provoque chez SaintOnge une véritable indignation, alors qu'elle dénonce le peu de place laissée aux écrivains classiques, au profit d'auteurs approuvés par le clergé, qu'elle considère comme mineurs. La dénonciation vient ici d'un certain recul de la femme adulte qui, plus tard, découvrira par exemple qu'Anatole France n'était pas ce "vil contempteur de Jeanne d'Arc " dont lui avaient parlé les auteurs de son manuel d'histoire littéraire. Claire Martin renchérit à ce propos et développe une réelle frustration par rapport au manuel qui limite sa lecture et met un frein à sa liberté : «Un jour, je serais libre et je pourrais lire la suite de tous ces extraits qu'on avait triés, me semblait-il, dans la seule intention de me faire enrager de curiosité ${ }^{14}$. " La réaction de Claire Martin, caractérisée par une violence plus marquée que chez ses consœurs et par un désir de se libérer du carcan étouffant de la prescription, est tout à fait évocatrice. Issue d'un milieu populaire et orpheline de mère à l'âge de 13 ans, Claire Martin subit l'autorité tyrannique d'un père abominable, qui interdit entre autres choses la lecture à ses enfants et particulièrement aux filles. Ainsi, Claire Martin ne possède que ce manuel scolaire pour découvrir la littérature et cherche à y assouvir une curiosité lésée à la maison. D'emblée, elle engage un combat marqué par la colère contre le moindre obstacle à sa liberté, contre les limites imposées par l'autorité associées à l'abus de pouvoir exercé par un père odieux.

L'accès aux auteurs classiques qui ne sont pas approuvés par le clergé se trouve donc limité pour les jeunes filles du corpus, mais la véritable contrariété face aux lectures scolaires concerne les manuels de morale et de religion. LesageVézina et Saint-Onge, toutes deux pensionnaires dans les 
années 1930-1940, font référence aux manuels de philosophie de l'abbé Arthur Robert, Leçons de logique, Leçons de psychologie et de théodicée et Leçons de morale, destinés aux élèves qui ne fréquentaient pas les collèges classiques ${ }^{15}$. Lesage-Vézina les commente en ces mots :

on y décelait la marque de l'enseignement didactique religieux avec des questions posées et des réponses imposées comme dans le petit catéchisme où l'apport de la curiosité et de la recherche individuelle était absent $^{16}$.

La visée morale et la valeur didactique de tels manuels deviennent pour certaines têtes lucides presque indigestes, alors que le livre impose une façon de penser et limite le savoir. Lesage-Vézina dénonce le fait que les enseignants visent uniquement l'instruction et non la réflexion individuelle dans ces manuels, et l'absence de stimulation intellectuelle devient l'objet de critiques persistantes chez elle.

Pour Paule Saint-Onge, fervente dénonciatrice dans son autobiographie des comportements irrationnels d'une mère hystérique et des affres d'un milieu pauvre et inculte, les manuels scolaires sont l'expression d'une mentalité arriérée autant présente dans sa famille qu'à l'école. Elle rappelle qu'Arthur Robert s'oppose dans son manuel de morale à "l'école obligatoire sous prétexte que les enfants pauvres, qui n'ont pas de quoi se chausser, seraient confrontés à une grave humiliation $^{17}$ " et que $\mathrm{M}^{\mathrm{gr}}$ Cauly, dans son Catéchisme, explique l'existence de Dieu selon le "consentement universel des peuples $^{18}$ ». Déjà enfant, elle reste "stupéfaite " face aux arguments de son "catéchiseur", profondément dégoûtée par les idées rétrogrades de ses précepteurs. Le didactisme des livres prescrits provoque chez elle une frustration qui sera le ferment d'une lutte qu'elle entreprend pour réussir à penser par elle-même, pour se libérer du joug d'un enseignement étroit : 
"J'étais fermement résolue à chercher ailleurs que dans Cauly des motifs sérieux pour continuer à croire ${ }^{19}$. " Déçue de l'enseignement scolaire, la jeune lectrice devient autonome, refusant d'être modelée par un didactisme réducteur et limitatif. Saint-Onge se rabat en effet sur ses propres ressources pour repérer des lectures plus stimulantes : «On nous passait des livres pieux d'une lecture éprouvante, mais j'arrivais parfois à dénicher parmi toute cette fadeur quelque biographie qui avait d'autres mérites que ceux de l'édification ${ }^{20}$. " La lectrice débrouillarde devient donc autodidacte et redéfinit son espace de lecture au-delà des limites imposées par la prescription, dans ce monde qu'elle conquiert seule pour mieux se l'approprier.

Outre le didactisme des lectures scolaires, la présence de livres à la maison fait l'objet de plusieurs commentaires dans les quatre autobiographies et semble influencer grandement la relation qui s'établit entre l'enfant et le livre. Selon ces témoignages, les lectures d'enfance et de jeunesse hors des couvents consistent, d'une part, en cette littérature morale formée principalement des contes de Perrault et de quelques histoires pour enfants éditées en France (la « Bibliothèque rose » de la comtesse de Ségur, les livres de la Maison de la Bonne Presse ou de la Maison Mame et fils, les histoires de Geneviève de Brabant, la série des Brigitte de Berthes Bernages, La Semaine de Suzette, etc.) et, d'autre part, en cette littérature religieuse qui prolifère dans les maisons québécoises, composée principalement de la vie des saints, des annales religieuses et de l'Évangile. La description des autobiographes dénonce tout d'abord l'univers mental inapproprié pour un enfant qui se dégage de ces livres moraux et religieux. La profusion d'images effrayantes de mort et de péché contribue manifestement à donner aux lectures enfantines un aspect lugubre et traumatisant. 
Lesage-Vézina s'attarde longuement sur l'influence d'un ouvrage qui s'intitule le Catéchisme en images ${ }^{21}$ et qui marque son imaginaire religieux dès l'enfance, alors qu'elle le consulte à l'âge où seules les images parlent. Dans ce livre destiné aux enfants, on trouve "un tableau de l'enfer plein de démons et de damnés ${ }^{22}$ ", de quoi stupéfier et effrayer l'enfant à peine familier avec l'idée de la mort. Lesage-Vézina dénonce cette première influence funeste et terrifiante de la religion par l'intermédiaire de ce contact prématuré avec un imaginaire morbide et une psychologie macabre. Elle déclare ensuite : "Au début du siècle, au Québec, la société était tournée vers son passé, maintenue par le clergé et le pouvoir dans un climat religieux d'ignorance et de peur. Tout à fait dans la lignée de mon Catéchisme en images ${ }^{23}$. " Lesage-Vézina dénonce clairement ce « maintien » de la société québécoise sous l'autorité religieuse, caractéristique du pouvoir prescriptif qui, comme un chien de garde, exerce un contrôle en délimitant l'espace de liberté par l'utilisation de la peur. Cependant, Lesage-Vézina jouit d'un soutien physique et moral privilégié dans la lecture qui la rassure face à cet univers effrayant : "En m'initiant très tôt à la lecture et au dialogue, tante $\mathrm{Ma}$ riette contrait du même coup les effets négatifs de certains tableaux que la vie multipliait sous nos yeux, et que j'avais prévus avec mon Catéchisme en images ${ }^{24}$. " L'importance du rôle de l'accompagnateur dans la lecture prend alors tout son sens. Selon le témoignage de Lesage-Vézina, l'enfant qui bénéficie d'un encadrement dans la lecture, soit d'un guide de lecture proche et disponible, réussit à se libérer de l'influence nocive d'une lecture inappropriée.

Saint-Onge insiste également sur la prépondérance du thème de la mort dans les livres de son enfance, mais contrairement à Lesage-Vézina, elle ne profite pas d'une présence humaine apaisante : 
Ainsi, assez curieusement, la plupart des rêves de mon enfance et de mon adolescence ont tourné autour du thème de la Mort, car si les livres m'avaient appris à bien mourir, personne ne m'avait enseigné l'art de vivre $^{25}$.

Il est intéressant de noter la complémentarité absolument nécessaire entre le livre et le guide de lecture, maintes fois relevée dans les textes du corpus. Ici, l'apprentissage offert par la lecture n'est pas éclairé par une lumière extérieure, soit un parent ou un mentor qui accompagne l'enfant dans son cheminement intellectuel. En ce sens, il est possible d'avancer l'idée que l'expérience individuelle de lecture, selon le « cadre affectif » dans lequel elle s'inscrit, fait varier la dynamique de lecture et influence grandement sa représentation. Le livre acquiert donc un éclairage pluriel grâce à l'analyse de la lecture dans l'autobiographie, car il est décrit selon la relation individuelle de chacun et selon les différents prismes qui l'éclairent. Il semble que les multiples perceptions du livre en disent encore plus long sur son histoire que son aspect strictement matériel.

Les lectures prescrites aux jeunes filles du corpus s'orientent également vers un type de littérature spécifiquement destinée au sexe féminin. Des romans "à l'eau de rose " et des brochures destinées aux futures mères de famille viennent alimenter une vision traditionnelle de la femme, contre laquelle les jeunes filles du corpus semblent se révolter. Saint-Onge juge que la série de Mame et fils et les livres de Berthe Bernages ont quelque chose de dangereux : «Je me suis demandé par la suite si ces lectures prétendument édifiantes n'avaient pas eu un effet sournoisement nocif en cultivant chez moi une tendance au masochisme ${ }^{26}$. Elle dit plus loin que la série des Brigitte encourageait les privations et les maternités fréquen- 
tes chez la femme et véhiculait ainsi une vision traditionnelle et arriérée de la femme.

Selon Lesage-Vézina, la vision traditionnelle décidait des lectures de l'enfant selon les sexes, alors qu'on faisait lire aux filles des « romans sentimentaux idéalisant leur rôle de reine du foyer ${ }^{27} »$. Elle s'indigne de la ségrégation entre les sexes dans les lectures prescrites et se demande : "Pourquoi [...] la femme ne pouvait-elle pas recevoir le même enseignement [que les garçons] au lieu d'être cantonnée aux travaux ménagers et à la littérature dite à l'eau de rose ? $^{28}$ » La prescription toucherait donc différemment les sexes à l'époque, ce qui peut expliquer la réaction outrée des jeunes filles du corpus d'autant plus révoltées qu'elles subissent une contrainte de lecture plus importante que les garçons de leur âge. L'expression "cantonnée à la littérature à l'eau de rose " représente cette idée tant évoquée d'une littérature spécifiquement féminine, douceâtre, fade et puérile, qui limite les possibilités de la femme et représente un obstacle de plus à l'obtention d'une liberté de lecture.

Chez Claire Martin, les lectures permises se restreignent aux biographies de saints, aux annales religieuses reçues gratuitement, aux bulletins paroissiaux, aux feuilletons de l'Action catholique et aux livres de prix reçus à l'école. Sa révolte contre le pessimisme et l'image réductrice de la femme qui se dégagent de ces brochures s'exprime sans détour: "L'esprit qui s'étalait dans ces brochurettes était propre à décourager n'importe quel humain d'exister et n'importe quelle femme d'être une femme ${ }^{29}$. » Limitée à des lectures abêtissantes et subissant l'humiliation constante d'un père misogyne, Claire Martin comprend très tôt que les seuls livres permis chez elle la maintiennent dans l'ignorance. Scandalisée, elle se révolte plus agressivement que d'autres jeunes filles chez qui le didactisme et le traditionalisme des lectures ne s'étendent pas 
à l'éducation familiale. La contrainte du livre lui renvoie l'image du parent despote qui l'empêche de penser, de vivre et de respirer librement. La prescription représenterait donc pour Claire Martin une loi à la fois sociale et familiale. Ainsi, la frustration par rapport à la loi de la prescription et de la proscription se fera plus viscérale chez elle, la souffrance se vivant à l'intérieur.

En revanche, pour Simonne Monet-Chartrand, élevée dans un milieu libéral où on dénonce la prescription et la proscription abusives, l'indignation envers le didactisme et le traditionalisme des livres prescrits se fait beaucoup plus contenue et réfléchie.

En regardant les pages couvertures et la page intérieure de tous les manuels imposés que j'ai étudiés, [écrit-elle] j'y vois la préséance et l'influence prépondérante de la religion catholique. D'abord par ses auteurs, tous des clercs. Ensuite par la mise en garde et la condamnation de toute idée moderne et l'imprimatur de l'évêque exigé lors de la publication de tout manuel scolaire, dans la province de Québec ${ }^{30}$.

Simonne Monet-Chartrand écrit ce commentaire dans son Journal (recopié dans son autobiographie) en 1937, alors qu'elle a 18 ans. Elle alimente visiblement sa révolte face à la vision religieuse imposée à la jeunesse québécoise du discours paternel, lui-même critique face au clergé. Simonne se trouve entre deux positions, soit celle de sa mère qui privilégie le rôle traditionnel de la femme mais qui subit aussi l'opinion de son mari, Amédée Monet, un juge libéral qui côtoie et encourage des idées modernes et une vision plutôt progressiste de la société, pour lesquels semble pencher la jeune fille. Face à la censure, elle jouit d'un milieu familial qui l'accompagne et l'oriente dans ses lectures: " mon milieu familial me surprotège, privilégie et favorise mes goûts pour l'étude et la 
culture. Je me sens bien reconnaissante envers les miens ${ }^{31}$ ", écrira-t-elle, pleinement consciente du privilège de sa situation de jeune bourgeoise. Sa dénonciation de la prescription diffère donc de celle des autres jeunes filles du corpus, parce qu'elle se nourrit du discours critique entendu chez elle plutôt que d'une opposition généralisée à l'autorité familiale et sociale comme c'est le cas pour Claire Martin, ou d'une tendance autodidacte, comme pour Saint-Onge.

Il existe visiblement une forme de gradation dans la critique du livre prescrit qui se révèle selon l'implication familiale des dénonciatrices. Pour une enfant comme Simonne Monet-Chartrand, la critique suit une ligne de pensée enseignée dans le milieu familial, lui-même hostile à la vision traditionaliste imposée par la société. Ainsi, la révolte se fait calme et raisonnée, en accord avec une forme de pensée héritée des éducateurs. La transmission du savoir passe inévitablement par les éducateurs, et les témoignages de nos autobiographes rendent compte de l'importance majeure de ces initiateurs à la lecture qui forment une seconde tutelle bienfaisante de lecture, contrairement à la tutelle prescriptive souvent limitative.

Chez Thérèse Lesage-Vézina, choyée par une tante disponible et une bibliothèque abondante, le didactisme des manuels scolaires et l'univers morbide et peu stimulant des livres pour enfants provoquent une indignation, mais l'accompagnatrice de lecture joue un rôle beaucoup plus fondamental. Orpheline très jeune, la petite Thérèse Lesage-Vézina sera secourue par sa marraine, qui l'initie à la lecture et lui fait découvrir sa fonction rédemptrice : "Je crois comprendre aujourd'hui que ma marraine (tante Mariette), ayant elle-même perdu sa mère à l'âge de dix ans, avait réalisé que les lectures pouvaient libérer une douleur muette ${ }^{32}$. " Le livre, accompagné par la marraine, devient «thérapeutique», pour employer 
son expression, parce qu'il comble un manque (la mère décédée) et qu'il exerce un rôle curatif face aux menaces extérieures. Cet accompagnement explique peut-être le profond attachement de la petite fille à la lecture et, peut-être, son moindre engagement contre une censure qu'elle ne vit pas de l'intérieur.

La prescription influencerait donc différemment les pratiques de lecture durant l'enfance selon le rapport à l'autorité et le degré de liberté vécus dans le milieu familial. Le livre occupe en effet une position problématique, à la fois soumis à des limites dictées par les prescripteurs, mais aussi représentatif d'une ouverture sur la connaissance, d'une forme de liberté pour l'enfant qui découvre l'étendue du savoir. La bibliothèque familiale et les accompagnateurs de lecture deviennent ainsi déterminants, car ils peuvent soit permettre de dépasser et de comprendre les lectures imposées, soit venir augmenter la contrainte sociale par une dynamique familiale opprimante. D'une tutelle contraignante représentée par la loi prescriptive, l'enfant doit en quelque sorte passer à une tutelle bénéfique, incarnée par des guides de lecture. L'autre versant de la censure, soit la proscription, semble répondre sensiblement à cette même logique, mais cette fois-ci, la dimension du rapport à l'autorité s'organise autour de l'interdit et non de la contrainte, ce qui vient bouleverser les jeunes lecteurs dans une toute autre mesure.

\section{Lectures d'enfance et de jeunesse proscrites ou les paradoxes de la retraite}

L'interdit génère des réactions et des dynamiques de lecture particulières que les autobiographies du corpus mettent en scène abondamment, probablement parce que ces pratiques de lecture engagent nos lectrices dans une forme de positionnement social et identitaire fondamental à leur che- 
minement de vie. Sans doute la censure n'a-t-elle pas toujours eu les mêmes effets sur la jeunesse, et le rapport face au livre interdit évolue très certainement selon une dynamique sociale elle-même changeante. Au XIX $\mathrm{X}^{\mathrm{e}}$ siècle, un exemple illustre une image de la censure qui semble bien éloignée de celle qui prévaut dans la première moitié du $\mathrm{XX}^{\mathrm{e}}$ siècle. Dans le Journal d'Henriette Dessaulles, jeune bourgeoise issue du milieu libéral de la fin du XIX $\mathrm{X}^{\mathrm{e}}$ siècle, il n'est presque pas question de l'interdit, sauf à une occasion. La scène tourne autour d'un livre non identifié, qu'un ami offre à Henriette, mais que son prétendant refuse qu'elle lise. Dessaulles commente la scène en ces mots :

Et voilà comment sont menées les petites filles dont l'ami est un Sage! En y pensant devant les étoiles, ce soir, je suis touchée de tout ce que sa sollicitude implique de délicatesse, de protection et de tendresse qui enveloppe la petite âme confiante et un peu craintive que je suis ${ }^{33}$.

En 1880, donc, la censure apparaît à cette jeune fille comme une protection bienfaisante et l'interdit se fait rassurant, peutêtre parce qu'elle n'a pas à se plaindre des livres à sa disposition, mais peut-être aussi parce qu'elle apprécie particulièrement cette autorité attentive à contrôler ses lectures qui la met à l'abri de livres dangereux ou non conformes à sa condition.

Cette seconde hypothèse suppose que la perception de l'interdit se transforme peu à peu entre la fin du XIX ${ }^{\mathrm{e}}$ siècle et le milieu du $\mathrm{XX}^{\mathrm{e}}$ siècle, en raison de l'évolution de la condition de la femme, qui remet en cause l'interdit de lecture dorénavant perçu comme un véritable obstacle au savoir, à une forme de liberté qu'elle commence à réclamer. Les réactions face à l'interdit dans les autobiographies du $\mathrm{XX}^{\mathrm{e}}$ siècle étudiées dans le cadre de cette analyse semblent en effet se lier à 
une nouvelle définition de l'identité féminine, mais aussi à cette nouvelle forme de " censure extensive » dont parle Pierre Hébert $^{34}$ moins monologique, intériorisée et disséminée dans des petits groupes sociaux moins visibles, mais tout aussi présente, sinon plus puissante.

La plupart des filles du corpus s'opposent à la proscription en la déjouant, mis à part Lesage-Vézina, qui n'accède à aucun livre interdit et affirme dans son autobiographie que le Québec de son enfance était loin du "droit à la dissidence ", apparu selon elle avec le Refus global en 1948. Pourtant, l'exemple de la sortie remarquée des Demi-civilisés de Jean-Charles Harvey $^{35}$ et les témoignages des autres jeunes filles du corpus illustrent une certaine tendance à la dissidence, contrairement à ce qu'avance Lesage-Vézina. Il y a sans doute un large fossé entre ce qui se passe officiellement et ouvertement dans la société québécoise des années 1930-40-50 et ce qui occupe les esprits, en retrait du monde et des lois officielles. La contestation prend des allures peut-être discrètes, mais elle existe, comme l'attestent les femmes du corpus. De plus, le témoignage de Lesage-Vézina reflète une réalité rurale, bien différente de celles de Claire Martin, Simonne Monet-Chartrand et Paule Saint-Onge, toutes trois Montréalaises. Pont-Rouge, le petit village de la région de Québec où grandit LesageVézina, n'offre pas d'accès aux livres mis à l'Index selon son affirmation. Celle-ci n'est donc pas consciente durant son enfance des effets néfastes de la proscription et encore moins des possibilités de la détourner.

Pour les trois autres filles, originaires de Montréal, la proscription, en plus d'être connue, sera déjouée et à la source de plusieurs conflits intérieurs. Ainsi, la lecture interdite devient un motif essentiel dans les autobiographies étudiées. Élevée contre l'autorité, cette lecture génère une série de mécanismes particuliers qui viennent divulguer une manière 
de lire singulière et décisive chez ces filles en pleine formation intellectuelle et personnelle. En retrait du monde, la lecture clandestine initie à une pratique dissidente mais aussi stimulante, comme le décrit Paule Saint-Onge :

Les obstacles et la censure ont toujours stimulé les mauvaises têtes et ne les ont pas empêchées d'explorer les mondes interdits. Ils ont seulement retardé le processus. Mais ces écritures et ces lectures secrètes et anodines n'ont été, au fond, que l'élément piquant dont une adolescente a besoin pour se prouver qu'elle existe. Assez curieusement, l'internat a été, pour moi, l'exploration d'une certaine libertés ${ }^{36}$.

Paule Saint-Onge évoque ici l'aspect profondément stimulant de l'interdit et le sentiment de liberté qui se dégage de sa transgression. La proscription semble aiguiser la curiosité de la lectrice rebelle qui découvre une forme d'affirmation de soi dans la violation de la loi. Il faut cependant rester prudent vis-à-vis de cette image de l'héroïne marginale qui lutte contre la Loi et regimbe contre toute forme d'autorité, car il s'agit d'une autobiographie et donc d'une représentation de soi. Les lectrices ont certainement mis l'accent sur cette caractéristique afin de projeter une image positive d'elles-mêmes. Il n'en demeure pas moins que l'interdit teinte leur relation à la lecture de manière évidente.

Cet aspect positif de la proscription habite également Claire Martin, pour qui la lecture clandestine s'accompagne d'une forte contestation de l'autorité paternelle. Arme d'opposition, le livre acquiert une valeur de combat, valeur toute symbolique qui vient en quelque sorte brouiller le jugement de la lectrice. En effet, parmi les livres interdits que se procure Claire Martin grâce à des amis ou cousins, la plupart sont jugés bons simplement parce qu'ils transgressent la Loi détestée : "Je trouvais tout beau, tout bien. Guy de Chantepleure, 
Mauriac, Zénaïde Fleuriot, Dekobra, Victor Marguerite et Delly, pour moi c'était tout pareil ${ }^{37} »$. La pratique secrète de la lecture crée chez Claire Martin une véritable idéalisation du livre et, en tant que lectrice débutante, elle ne connait pas les codes qui différencient la littérature légitimée de la littérature populaire. Évidemment, les livres interdits ne sont pas toujours de grande qualité, mais ils viennent avant tout combler un manque chez elle et se consomment frénétiquement :

La passion des livres, comme toutes les passions, se nourrit de succédanés quand elle n'a pas de vraie nourriture. L'ivrogne mal ravitaillé boit son eau de Cologne. J'ai fait une extraordinaire consommation d'eau de Cologne ${ }^{38}$.

La proscription doublée de l'interdit paternel crée donc chez elle une stimulation extrême pour la lecture, mais aussi une consommation livresque abondante et peu orientée. Le « mauvais ravitaillement " caractérise la lecture proscrite, souvent faite au hasard et avec des moyens restreints. L'image récurrente de la lectrice qui se "gave» de livres interdits renvoie à cette idée d'une pratique abusive et démesurée, qui vise à remplir un vide plutôt qu'à nourrir intelligemment.

Le paradoxe de la proscription se situe peut-être dans cet aspect contradictoire de la lecture clandestine, à la fois stimulante, libératrice, mais aussi déséquilibrée et réductrice parce qu'isolée du reste du monde. Claire Martin affirme que jusqu'à 25 ans, elle boit tout ce que disent les auteurs interdits, probablement par esprit de contradiction, adorant tout ce que la société condamne : «à vingt-cinq ans j'était fasciste et antisémite [...] La lecture de Gide suffisait à faire pencher à gauche et celle de Maurras à droite ${ }^{39}$ ». La conquête d'une certaine liberté par la lecture clandestine confine donc cette lectrice à une pensée qui n'est pas encore orientée, fruit d'un 
isolement intellectuel qui la prive de ressources suffisantes pour comprendre, expliquer et juger les livres lus.

Chez Paule Saint-Onge, la proscription se présente également comme une forme de stimulation équivoque. Elle consacre un chapitre à la découverte de la lecture et particulièrement aux livres lus en cachette, dont l'influence sur les gens de sa génération a été majeure selon ses dires. Évoquant deux livres interdits à la maison, Roméo et Juliette et L'école des amants, elle explique le mélange de curiosité et de culpabilité ressentie à la lecture de ces objets tabous. Pour camoufler l'acte illicite, la petite Saint-Onge raconte qu'à l'âge de dix ans, elle dissimule Roméo et Juliette derrière un livre pieux, De la nécessité de la prière, pour ne pas être surprise en plein délit. Or les félicitations imméritées de son père sur sa soi-disant lecture vertueuse lui causent une honte insoutenable, la plongent dans un état d'ambivalence entre la fierté d'avoir réussi à déjouer l'autorité et l'extrême humiliation face à la trahison envers son père. Elle affirme que « quand tout est interdit à tort et à travers, on acquiert une curiosité aiguë et une certaine habileté à la satisfaire, et à cause de cette habileté, on se sent encore plus coupable ${ }^{40}$ ». Finalement, la transgression de la loi semble provoquer chez elle une sorte de mécanisme infernal qui mêle satisfaction et culpabilité, curiosité et répulsion, désir et frustration.

À la lecture de L'école des amants, le brouillage s'accentue d'autant plus que le contenu paraît véritablement douteux. Après avoir ressenti un mélange de plaisir et de répugnance à la lecture du livre, elle le jette au feu, comme pour se purger de la faute. Le livre devient donc à la fois un objet de désir et un objet de péché, une source de sentiments contradictoires qui viennent bouleverser la logique morale et psychologique de la jeune lectrice démunie face à l'interprétation d'un livre tant désiré mais plutôt décevant. Encore une 
fois, la liberté acquise par la lecture interdite souffre de l'isolement physique et intellectuel qui la caractérise et qui engage le lecteur dans un double mouvement de libération et de claustration. L'enfant qui s'isole pour lire et enfreint ainsi les règles de la proscription se retrouve effectivement dans une position problématique face à la société : il conteste la loi mais dans la solitude et donc en-dehors des lieux de débats réels, dans un espace intérieur, à l'écart du monde et propre à la lecture.

Les autobiographes du corpus évoquent tour à tour la découverte de la lecture comme une plongée intérieure dans un monde qui leur appartient, mais également comme une tentative de s'approprier le monde extérieur en contestant ses lois. La lecture peut en effet être perçue comme une prise de possession de soi et du monde, et elle prend ainsi tout son sens dans l'autobiographie comme étape charnière dans la formation de l'identité. Même Simonne Monet-Chartrand, qui ne souffre pas de la proscription chez elle, recherche une forme d'isolement dans la lecture, signe d'une certaine indépendance, comme si l'affirmation de soi devait passer par une lecture autonome. Dans son journal de 1937, alors qu'elle a 17 ou 18 ans, elle déclare:

J'éprouve une fringale de lecture. Je ne fais que lire je fuis tout le monde - je vis dans un autre monde, même plus, dans une autre époque. [...] Excellente raison de n'avoir pas à subir les mours de mon milieu «bourgeois ». J'en suis libérée. Pas d'activités «mondaines ». J'expérimente une fuite, fuite de la réalité je l'admets, mais combien salutaire pour $\mathrm{moi}^{41}$.

La fuite que revendique la petite Simonne représente l'indépendance positive de l'enfant qui découvre dans la lecture ses possibilités d'évasion. Cependant, l'indépendance peut devenir contraignante pour le lecteur qui se coupe du reste du 
monde et subit la proscription comme un obstacle à l'ouverture sur les autres. Les témoignages de Claire Martin et de Paule Saint-Onge illustrent les limites de cet isolement de la lecture clandestine. Il serait donc possible d'affirmer que la proscription crée parfois un climat de désocialisation où errent des solitudes qui contestent dans le silence et ne peuvent joindre leurs luttes.

D'où cette importance relevée précédemment des accompagnateurs de lecture et des rencontres extérieures pour éclairer les livres des connaissances du groupe et pour intégrer l'individu à la société. Les femmes de notre corpus évoquent la difficulté causée par le manque de contacts propre au climat de la proscription et chantent la vertu des rencontres fructueuses autour d'un livre. Une religieuse enseignante offre par exemple à Saint-Onge le poème «L'idiote aux cloches » de Nelligan, événement considéré comme une véritable rédemption pour la petite fille esseulée.

À cette époque, dans le milieu modeste et inculte qui était le mien, j'aurais pu, sans elle [la religieuse], attendre des années avant de faire la rencontre bouleversante de la Beauté si étroitement liée au tragique. Ce sont de telles rencontres qui changent le cours d'une $v^{4} e^{42}$.

La rencontre libère donc la lectrice de son isolement, qui devient à la longue douloureux et stérile intellectuellement. L'exemple de Claire Martin, qui par son extrême désir de contester l'autorité dévore une littérature de masse, illustre parfaitement les limites de l'intériorisation créée par la proscription. Au fur et à mesure, le besoin de l'autre se fait pressant pour la plupart des jeunes filles du corpus, l'interlocuteur devenant crucial pour que la contestation porte fruit.

L'expérience de Simonne Monet-Chartrand, qui bénéficie du dialogue et d'une visibilité sociale grâce aux groupes 
de pression auxquels elles participe, révèle les débuts de la lutte ouverte contre la censure au Québec et l'étape ultérieure dans la revendication d'une liberté de penser. Alors que Claire Martin et Paule Saint-Onge luttent contre la proscription en lisant en cachette des livres interdits, Simonne MonetChartrand franchit un pas de plus en portant le combat de l'espace intérieur, propre à la lecture, à l'espace public, propre à la discussion et garant de l'avancement social. Elle rapporte une conversation avec son père, suite à un article dans le journal portant sur la censure ecclésiastique. Le père de Simonne explique les abus de pouvoir du clergé dans la publication d'œuvres littéraires et défend les Demi-civilisés d'Harvey. Il affirme qu'il laisserait Simonne lire ce livre s'il était publié à Montréal : "C'est à toi de te servir de ton jugement. C'est ça le libre-arbitre ${ }^{43}$. " Simonne apprend donc à juger par elle-même et à choisir ses lectures librement, sans souffrir de l'isolement qui cantonne le lecteur à une incompréhension et un combat unidirectionnel avec lui-même. Elle jouit plutôt du dialogue qui prévaut chez elle.

Sa collaboration à la Jeunesse étudiante catholique (JEC) lui permet également d'unir ses forces à d'autres jeunes. Grâce à cette organisation, elle découvre des auteurs modernes, une spiritualité laïque et l'avantage de s'associer dans la lecture :

À la Centrale, grâce aux contacts internationaux des dirigeants et des aumôniers de la JEC, nous avons eu la chance de lire des auteurs à la fine pointe de la spiritualité laïque. Nous échangions nos vues et commentaires "en équipe » sur ces poètes, philosophes ou auteurs spirituels ${ }^{44}$.

Ces auteurs mis à la marge de l'orthodoxie et lus à la JEC sont Charles Péguy, Jacques Maritain, Jacques Rivière, Léon Bloy, Georges Bernanos et quelques autres, dont la lecture ne peut profiter à une jeunesse isolée. À ce titre, l'échange devient 
essentiel à la compréhension et à la revendication d'une pensée nouvelle, forcément dépendante d'une unification des forces.

Il peut paraître paradoxal que des groupes chrétiens soient les premiers lieux où la jeunesse québécoise sorte de son isolement pour combattre la censure et l'autorité cléricale. Simonne Monet-Chartrand évoque cependant très clairement ses intentions lorsqu'elle s'engage dans l'Action catholique :

Moi, j'ai d'autres raisons de militer dans l'Action Catholique. C'est comme fille majeure, laïque et instruite de mes responsabilités, consciente de la bonne influence que je peux exercer pour apprendre aux filles à réfléchir ; à parler tout haut, à se grouper ${ }^{45}$.

Cette jeune fille sûre d'elle-même représente la première génération de femmes qui, après la crise, briseront le mur du silence pour enfin dire tout haut ce qu'elles pensaient tout bas, dans le retrait de ces lectures cachées qui, en quelque sorte, ont peut-être constitué le ferment des luttes contre la censure, du combat pour la liberté de penser. La Révolution tranquille, initiée par la génération de ces femmes qui avaient entre 35 et 45 ans en 1960, est souvent associée à la fin d'une "grande noirceur». Dans le domaine de la lecture, la fin d'un profond isolement a sans doute marqué bon nombre de lecteurs et lectrices, dont l'identité devait dorénavant s'extraire des contraintes du secret, limitatif et peu fertile à l'intégration sociale.

\section{$* * *$}

Au terme de cette étude, il est possible d'affirmer que la contrainte et la répression dans le domaine du livre ont façonné les lectrices du corpus de manière significative, et ce, jusque dans la constitution de leur identité et de leur engage- 
ment social. Majoritairement écrasant, l'interdit peut aussi avoir un effet positif, en ce qu'il stimule et sollicite l'action et la lutte pour la conquête d'une liberté, mais il demeure néanmoins un joug duquel il faut s'extraire. La problématique de la censure du livre dans l'histoire des lectures de jeunesse se caractérise par deux aspects fondamentaux : une plongée intérieure que suppose la lecture secrète, liée à l'affirmation de soi et à la revendication d'une liberté de penser, et la découverte de l'importance majeure de l'extérieur, du besoin de l'interlocuteur pour nourrir un débat fleurissant, pour unir sa parole à celle de ses pairs. Ce double mouvement vers soi et vers l'autre caractérise la recherche identitaire, qui peut expliquer la présence abondante des lectures de jeunesse dans le genre de l'autobiographie.

Pour comprendre la singularité de la pratique de lecture, l'autobiographie semble constituer une source documentaire intéressante, qui révèle la particularité de la relation de l'individu au livre et offre un témoignage précieux pour l'histoire des mentalités. Philippe Lejeune note d'ailleurs l'importance du rôle de la lecture dans l'autobiographie, qui est souvent une phase initiatique dans la formation de l'identitét ${ }^{46}$. Jean Starobinski a découvert dans Les Confessions de Rousseau que les premières lectures coïncident avec la conscience de soi initiale, étroitement liée à la découverte de l'imaginaire qui permet de devenir un autre, de sortir de soi-même ${ }^{47}$. Ces réflexions sur le rôle de la lecture dans l'histoire d'une vie nécessitent un certain recul dans le temps, une perspective globale que permet le genre de l'autobiographie, contrairement au genre de la correspondance, qui pose un regard immédiat sur la réalité. Le phénomène de la lecture jouit également d'une place de choix dans l'autobiographie, parce que le livre coïncide bien souvent avec un point tournant de l'existence, avec cet éveil de la conscience individuelle dont parle 
Starobinski à propos de Rousseau. Entre le silence et l'isolement propres à lecture et la rencontre générée par le livre, l'enfant explore cet espace particulier de la pensée, à la fois muette et dialogique, intérieure et propulsée vers l'autre. Façonnant la représentation de soi et du monde, la lecture constitue donc un objet primordial du genre autobiographique, en tant qu'activité qui engage l'être vers les autres et initie à la conquête du monde, mais qui offre également une forme d'indépendance et d'autonomie intellectuelles, une délimitation de l'espace de soi.

En définitive, la lecture constitue une des premières formes de liberté revendiquée par l'individu, et ce dès l'enfance, car elle est un tremplin vers le dialogue et confronte avec une pensée extérieure, interdite ou obligatoire, de laquelle l'enfant se distancie ou à laquelle il s'identifie. Ainsi, l'analyse des perceptions intérieures de la lecture dans l'autobiographie révèle les rapports à l'autorité et les premiers pas de l'affirmation de soi, et renseigne autant sur l'état du livre que sur l'histoire du combat pour une liberté de penser, pour un droit à l'indépendance d'esprit. Par l'analyse des réactions intérieures des jeunes lectrices du corpus, ce sont les relations à de multiples figures d'autorité qui se révèlent, ces acteurs réels ou symboliques qui déterminent la relation de lecture et délimitent un espace plus ou moins limité d'apprentissage. Pour comprendre la lecture selon la perspective individuelle, il faut donc définir la chaîne des relations entre le lecteur et les formes d'autorité qui exercent une pression plus ou moins importante à son égard et dessiner cet espace intérieur du lecteur qui apprend à se situer face à l'autre. La variété des formes d'autorité évoquées dans cette étude révèle la complexité du combat identitaire de l'enfant, à la fois avide de se séparer d'une autorité du parent et du censeur, mais ayant besoin d'initiateurs et d'accompagnateurs dans son processus 
d'affirmation de soi. L'instituteur, le "passeur» de livres ou les pairs sont alors essentiels pour s'identifier à un "autre» positif et les jeunes filles ont particulièrement besoin de trouver des modèles féminins, tant dans leurs lectures que parmi les femmes qui les guident dans leur instruction. Simonne Monet-Chartrand offre aux femmes une première « voix » dans la société et la liberté de lecture constitue une première étape vers la libre intégration dans le monde.

Il existe aussi des formes d'autorités symboliques qui font pression sur nos lectrices, telles les images du péché, de la mort et de l'enfer, liées au climat de peur généré par l'interdit. En fait, l'histoire de la lecture de jeunesse se présente comme le récit de la formation de l'imaginaire individuel d'après un positionnement par rapport à un réseau d'autorités appartenant à l'imaginaire collectif, auxquelles l'individu se lie ou s'oppose, selon qu'elles se présentent comme bénéfiques ou menaçantes. Ainsi, la lecture constitue un phénomène à la fois individuel et social qui, dans le cas des femmes du corpus, correspond, d'une part, à une prise de conscience du pouvoir de l'autorité dominante et, d'autre part, à une volonté d'acquérir l'autonomie nécessaire pour exprimer ses opinions et défendre ses droits dans la société.

À travers l'histoire de la lecture dissidente et libératrice des jeunes filles du corpus, s'esquisse donc le parcours de la lutte identitaire des femmes de l'époque, qui s'élèvent contre une norme à la fois prescriptive et proscriptive et se définissent à partir d'une dynamique censoriale très forte, oppressante mais aussi stimulante. Il semble que le livre soit beaucoup plus un objet de liberté et la lecture une activité thérapeutique dans le contexte censorial de l'époque qu'il ne peut l'être aujourd'hui, bien que notre soi-disant libéralisme intellectuel dissimule encore des interdits et que la levée totale de la censure soit impossible et illusoire. La conquête pour une 
lecture autonome, enrichissante et libre, constitue toutefois une étape primordiale dans l'histoire de la libération des esprits des Québécois et des Québécoises à la moitié du $\mathrm{XX}^{\mathrm{e}}$ siècle, mais également dans l'histoire de l'émancipation des femmes qui utilisent le livre comme arme de délivrance.

\section{NOTES}

1 Paule Saint-Onge, La vie défigurée, Montréal, Éditions La Presse, 1979, p. 73.

${ }^{2}$ Yvan Lamonde et Sophie Montreuil, dir., Lire au Québec au XIX siècle, Montréal, Fides, 2004, p. 8.

${ }^{3}$ Parmi ceux qui se sont intéressés au rôle des sources personnelles dans l'histoire du livre et de la lecture, il faut retenir : James Raven, Helen Small et Naomi Tadmor dans leur introduction à l'ouvrage The Practice and Representation of Reading in England, Cambridge, Cambridge University Press, 1996, pp. 121 ; Robert Darnton, «First Steps Toward a History of Reading ", dans The Kiss of Lamourette: Reflections in Cultural History, New York, W.W. Norton, 1990, pp. 155-187 ; James Raven, « New Reading Histories, Print Culture and the Identification of Change: The Case of Eighteenth-Century England", Social History, vol. XXIII, $\mathrm{n}^{\circ} 3$ (octobre 1998), pp. 268-287 ; Frédéric Barbier, "L'Histoire, L'Historien et la Lecture », Gutenberg-Jabrbuch, n" 73, (1998).

${ }^{4} \ll$ it is necessary to read the vestiges of actual reading experiences which we find in diaries, autobiographies, letters and marginalia, in the light of an understanding of the concerns and the practices of the communities of readers to which their authors belonged. [...] Thus, the full significance of the traces of individual readings only becomes apparent when examined in the light of the reading practices of that reader's community. ", Jonathan R. Topham, "Scientific Publishing and the Reading of Science in NineteenthCentury Britain: A Historiographical Survey and Guide to Sources », Studies in History and Pbilosopby of Science, vol. 31, $\mathrm{n}^{\circ} 4$ (2000), pp. 571-572.

${ }^{5}$ Roger Chartier note l'enjeu suscité par les sources personnelles : « how to organize this indistinguishable plurality of individual acts according to shared regularities ? " ("Texts, Printing, Readings », dans Lynn Hunt, dir., The New Cultural History: Essays, Berkeley et Londres, University of California Press, 1989, p. 156.) James Raven répond à cette question dans des termes opportuns : «An answer implicit in recent studies is to downplay the importance 
of broad uniformities and to focus instead upon the qualitative differences of experience. » (" New Reading Histories, Print Culture and the Identification of Change: The Case of Eighteenth-Century England ", Social History, vol. 23, no 3 [octobre 1998], pp. 271.)

'Les quatre autobiographies ont été sélectionnées parmi un corpus de dixneuf textes de la littérature personnelle québécoise écrite par des femmes aux $\mathrm{XIX}^{\mathrm{e}}$ et $\mathrm{XX}^{\mathrm{e}}$ siècles, répertoriés dans deux ouvrages : Yvan Lamonde, Je me souviens : La littérature personnelle au Québec (1860-1980), Québec, Institut québécois de recherche sur la culture, 1983, coll. «Instruments de travail », $\mathrm{n}^{\circ} 9$, 275 p. ; Yvan Lamonde et Marie-Pierre Turcot, La littérature personnelle au Québec (1980-2000), Montréal, Bibliothèque nationale du Québec, 2000, 100 p. Cette recherche a été menée à l'Université McGill dans le cadre d'une étude qui s'intègre au projet « History of the Book in Canada / Histoire du livre et de l'imprimé au Canada ", subventionné par le programme des Grands travaux de recherche concertée du Conseil de recherches en sciences humaines du Canada. La recherche a été menée simultanément sur deux corpus et Cécile Facal s'est penchée sur le cas des écrivains et intellectuels québécois du $\mathrm{XX}^{\mathrm{e}}$ siècle (texte à paraitre). Ces autobiographies sont les suivantes : Claire Martin, Dans un gant de fer, Montréal, Cercle du Livre de France, 1965, 235 p. La joue droite, Montréal, Cercle du Livre de France, 1966, 209 p ; Simonne MonetChartrand, Ma vie comme rivière, Montréal, Éditions du Remue-ménage, tome 1, (1919-1942), 1981, 296 p. ; tome 2, (1939-1949), 1982, 360 p. ; tome 3, (1949-1963), 1988, 341 p. ; tome 4, (1963-1992), 1992, 377 p ; Paule SaintOnge, La vie défigurée, Montréal, Éditions La Presse, 1979, 198 p ; Thérèse Lesage-Vézina, Les livres qui ont coloré ma vie : Récit autobiographique, Québec, Éditions TLV, 1998, $253 \mathrm{p}$.

${ }^{7}$ Philippe Lejeune, Le pacte autobiograpbique, Paris, Seuil, 1996 [1975], 382 p. (Coll. « Poétique »).

" « La croix et l'ordre : le clergé et la censure de l'imprimé au Québec », Documentation et bibliothèques, vol. 41, $\mathrm{n}^{\circ} 1$ (janvier-mars 1995),pp. 21-29; Censure et littérature au Québec : Le livre rrucifié, 1625-1919, Montréal, Fides, 1997, 290 p. ; "La censure 1920-1960", Voix et images, vol. 13, n² (hiver 1998) (dossier spécial présenté par Pierre Hébert),pp. 217-423; Censure et littérature au Québec : Des vieux couvents au plaisir de vivre, 1920-1959, Montréal, Fides, 2004, 252 p.

${ }^{9}$ "La censure 1920-1960", Voix et images, vol. 13, nº 2 (hiver 1998), p. 221.

${ }^{10}$ Ibid., p. 223. 
${ }^{11}$ Denise Escarpit, La litterature d'enfance et de jeunesse, Paris, PUF, 1981, p. 4.

${ }^{12}$ Thérèse Lesage-Vézina, Les livres qui ont coloré ma vie : Récit autobiographique, $\mathrm{p}$. 109.

${ }^{13}$ Paule Saint-Onge, La vie défigurée, p. 75.

${ }^{14}$ Claire Martin, La joue droite, p. 86.

${ }^{15}$ Le premier collège classique pour filles au Québec a vu le jour à Montréal en 1908. Aucune des femmes du corpus n'a fréquenté un collège classique.

${ }^{16}$ Thérèse Lesage-Vézina, Les livres qui ont coloré ma vie : Récit autobiographique, $\mathrm{p}$. 139.

${ }^{17}$ Paule Saint-Onge, La vie défigurée, p. 75.

${ }^{18}$ Ibid.

${ }^{19}$ Ibid., p. 76.

${ }^{20}$ Ibid., p. 79.

${ }^{21}$ Paris, Maison de la Bonne Presse, 1912.

${ }^{22}$ Thérèse Lesage-Vézina, Les livres qui ont coloré ma vie : Récit autobiographique, $\mathrm{p}$. 17.

${ }^{23}$ Ibid., p. 49.

${ }^{24}$ Ibid., p. 76.

${ }^{25}$ Paule Saint-Onge, La vie défigurée, p. 58.

${ }^{26}$ Ibid., p. 57.

${ }^{27}$ Thérèse Lesage-Vézina, Les livres qui ont coloré ma vie : Récit autobiographique, p. 140.

${ }^{28}$ Ibid., p. 141. C'est moi qui souligne.

${ }^{29}$ Claire Martin, La joue droite, p. 132.

31) Simonne Monet-Chartrand, Ma vie comme rivière, tome 1, (1919-1942), p. 206.

${ }^{31}$ Ibid., Journal, 29 mars 1937, [sans pagination] (inséré dans l'autobiographie).

${ }^{32}$ Thérèse Lesage-Vézina, Les livres qui ont coloré ma vie : Récit autobiographique, $\mathrm{p}$. 39. 
${ }^{33}$ Fadette. Journal d'Henriette Dessaulles (1874-1880), Montréal, Presses de l'Université de Montréal, 1989, $2^{\mathrm{e}}$ édition, p. 585. (Coll. «Bibliothèque du Nouveau Monde »).

${ }^{34}$ "La croix et l'ordre : le clergé et la censure de l'imprimé au Québec », Documentation et bibliothèques, vol. 41, $\mathrm{n}^{\circ} 1$ (janvier-mars 1995),pp. 21-29; « La censure 1920-1960 », Voix et images, vol. 13, nº 2 (hiver 1998),pp. 217-423.

${ }^{35}$ Montréal, les Éditions du Totem, 1934.

${ }^{36}$ Paule Saint-Onge, La vie défigurée, p. 73.

${ }^{37}$ Claire Martin, La joue droite, p. 133.

${ }^{38}$ Ibid., p. 139.

${ }^{39}$ Ibid., p. 134.

40 Paule Saint-Onge, La vie défigurée, p. 57.

${ }^{41}$ Simonne Monet-Chartrand, Ma vie comme rivière, tome I, p. 194.

${ }^{42}$ Paule Saint-Onge, La vie défigurée, p. 60.

${ }^{43}$ Simonne Monet-Chartrand, Ma vie comme rivière, tome I,pp. 235-237.

${ }^{44}$ Ibid., p. 235.

${ }^{45}$ Ibid., p. 226.

${ }^{46}$ Le pacte autobiographique, Paris, Seuil, 1996 [1975], 382 p. (Coll. « Poétique »).

${ }^{47}$ Jean-Jacques Rousseau : La transparence et l'obstacle, Paris, Gallimard, 1971, 457 p. 